\title{
ORDINARY DIFFERENTIAL INEQUALITIES AND QUASIMONOTONICITY IN ORDERED TOPOLOGICAL VECTOR SPACES
}

\author{
ROLAND UHL
}

(Communicated by Hal L. Smith)

\begin{abstract}
A well known comparison theorem on ordinary differential inequalities with quasimonotone right-hand side $f(t, x)$ was carried over by Volkmann (1972) to (pre)ordered topological vector spaces. We prove that the quasimonotonicity of $f$ is a necessary condition here if $f$ is continuous. Then it is shown that quasimonotonicity can be verified by considering only a few positive continuous linear functionals in the definition (for instance in $\ell_{\infty}$ by taking coordinate functionals).
\end{abstract}

\section{INTRODUCTION}

Quasimonotonicity has its origins in initial value problems

$$
u\left(t_{0}\right)=x_{0}, \quad u^{\prime}(t)=f(t, u(t)) \quad\left(t_{0} \leq t \leq t_{1}\right)
$$

and corresponding differential inequalities, when certain theorems were carried over from the scalar case to problems in $\mathbb{R}^{n}$. First, Müller (1927) [3] proved the existence of a solution to (1) between given lower and upper solutions, and Kamke [1] established extremal solutions. They referred to the componentwise ordering in $\mathbb{R}^{n}$, and they assumed that, roughly speaking, each component $f_{i}\left(t, x_{1}, \ldots, x_{n}\right)$ is monotone increasing in every $x_{j}$ with $j \neq i$. Later on, Walter [12] called such functions quasimonotone increasing in $x$. In some applications the term cooperative is used.

Finally, Volkmann (1972) [8] gave the general definition of quasimonotonicity in topological vector spaces preordered by a cone, which makes use of the dual cone. He carried over a very useful comparison theorem on ordinary differential inequalities where the right-hand side $f(t, x)$ is quasimonotone increasing in $x$. Our purpose is to prove that, conversely, the quasimonotonicity of $f$ is a necessary condition here in case $f$ is continuous. Then we will show that quasimonotonicity can be verified by considering only some small subset of the dual cone.

A generalization of Müller's theorem to preordered Banach spaces is given in [7]. Note that (1) need not have any local solution if $f$ is only assumed to be continuous and bounded. It should be pointed out that in some ordered Banach spaces the quasimonotonicity of $f$ implies the existence of solutions to (1), in fact extremal solutions, without any Lipschitz or compactness condition. Some of these results

Received by the editors December 10, 1996.

1991 Mathematics Subject Classification. Primary 34G20, 34A40, $47 \mathrm{H} 07$.

Key words and phrases. Quasimonotonicity, ordinary differential inequalities, comparison or monotonicity theorems, lower and upper solutions, ordered topological vector spaces. 
are based on the fixed point theorem of Lemmert [2]. See the survey [10]. Finally, quasimonotonicity is also significant for systems of parabolic differential equations, cf. [6], [12], and for fixed points of discontinuous functions, cf. [4].

\section{Notations}

Let $E$ be a Hausdorff topological vector space, and let $K \subset E$ be a cone (i.e. $K$ is closed, convex, nonempty, and satisfies $\lambda x \in K$ for all $\lambda \geq 0, x \in K)$. By the definition

$$
x \leq y \Longleftrightarrow y-x \in K \quad(x, y \in E),
$$

a preordering (a reflexive transitive relation) on $E$ is given; this preordering is an ordering (also antisymmetric) iff $K$ is strict (i.e. $x,-x \in K \Longrightarrow x=0_{E}$ ). Now $E$ is said to be preordered or ordered, respectively. We write

$$
x \ll y \Longleftrightarrow y \gg x \Longleftrightarrow y-x \in \operatorname{Int} K \quad(x, y \in E),
$$

where Int $K$ denotes the interior of $K$. The dual cone of $K$ is

$$
K^{*}=\left\{\varphi \in E_{\mathbb{R}^{*}}^{*} \varphi(x) \geq 0 \text { for all } x \in K\right\},
$$

where $E_{\mathbb{R}}^{*}$ denotes the dual of $E_{\mathbb{R}}$ ( $E$ regarded as a real space).

A function $f: G \rightarrow E$ on $G \subset E$ is said to be quasimonotone increasing if for all $x, y \in G$ and all $\varphi \in K^{*}$ the implication

$$
x \leq y, \varphi(x)=\varphi(y) \Longrightarrow \varphi(f(x)) \leq \varphi(f(y))
$$

holds. Finally, (P) will denote the following property, where now $f: D \rightarrow E$ has domain $D \subset \mathbb{R} \times E$.

(P) If $v, w:\left[t_{0}, t_{1}\right] \rightarrow E\left(t_{0}<t_{1}\right)$ are any differentiable functions such that $\operatorname{graph} v, \operatorname{graph} w \subset D, v\left(t_{0}\right) \ll w\left(t_{0}\right)$ and

$$
v^{\prime}(t)-f(t, v(t)) \ll w^{\prime}(t)-f(t, w(t)) \quad\left(t_{0} \leq t \leq t_{1}\right),
$$

then $v(t) \ll w(t)$ for $t_{0} \leq t \leq t_{1}$.

\section{3. (P) IMPLIES QUASIMONOTONICITY}

Roughly speaking, property $(\mathrm{P})$ and quasimonotonicity are equivalent.

Theorem 1. Let $E$ be a Hausdorff topological vector space preordered by a cone $K$ with Int $K \neq \emptyset$. Suppose $D \subset \mathbb{R} \times E$ is such that for every $(t, x) \in D$ there exist $\varepsilon>0$ and a neighborhood $G$ of $x$ satisfying $[t, t+\varepsilon) \times G \subset D$. Assume $f: D \rightarrow E$ is continuous. Then $(\mathrm{P})$ holds if and only if for each $t \in \mathbb{R}$ the function $x \mapsto f(t, x)$ is quasimonotone increasing.

Note that the quasimonotonicity of $f$ in $x$ always implies (P), by Volkmann [8], without any assumptions on $D$ and $f$. Simon and Volkmann [5] prove the converse in case $E$ is a Banach space. Here we give a proof for the general case, which is nevertheless much shorter.

Proof. Assume (P). Suppose $\left(t_{0}, x\right),\left(t_{0}, y\right) \in D$ and $\varphi \in K^{*}$ satisfy

$$
x \leq y, \quad \varphi(x)=\varphi(y) .
$$


Fix any $p \in \operatorname{Int} K$. For each $t_{1} \geq t_{0}$, define $v, w:\left[t_{0}, t_{1}\right] \rightarrow E$ by

$$
\begin{aligned}
v(t) & =x+\left(t-t_{0}\right)\left(f\left(t_{0}, x\right)-p\right), \\
w(t) & =y+\left(t_{1}-t_{0}\right) p+\left(t-t_{0}\right)\left(f\left(t_{0}, y\right)+p\right)
\end{aligned}
$$

$\left(t_{0} \leq t \leq t_{1}\right)$. Choosing $t_{1}>t_{0}$ sufficiently close to $t_{0}$, we may assume graph $v$, graph $w \subset D$, and

$$
\begin{aligned}
& f(t, v(t))-f\left(t_{0}, x\right)+p \in \operatorname{Int} K \\
& f\left(t_{0}, y\right)+p-f(t, w(t)) \in \operatorname{Int} K
\end{aligned}
$$

for $t_{0} \leq t \leq t_{1}$. For the left sides may be regarded as continuous functions of $\left(t_{1}, t\right)$, which assign $p$ to $\left(t_{0}, t_{0}\right)$. Thus we have

$$
v^{\prime}(t) \ll f(t, v(t)), \quad w^{\prime}(t) \gg f(t, w(t)) \quad\left(t_{0} \leq t \leq t_{1}\right),
$$

hence (3), and clearly $v\left(t_{0}\right) \ll w\left(t_{0}\right)$. By $(\mathrm{P})$, this implies $v\left(t_{1}\right) \leq w\left(t_{1}\right)$. We conclude that $\varphi\left(v\left(t_{1}\right)\right) \leq \varphi\left(w\left(t_{1}\right)\right)$, hence

$$
\varphi\left(f\left(t_{0}, x\right)-p\right) \leq \varphi\left(f\left(t_{0}, y\right)+2 p\right),
$$

and, letting $p \rightarrow 0_{E}, \varphi\left(f\left(t_{0}, x\right)\right) \leq \varphi\left(f\left(t_{0}, y\right)\right)$. Thus the quasimonotonicity of $f$ is verified.

Remark 1. If we replace (3) by (4) in (P) then Theorem 1 still holds, by the preceding proof. Moreover, if the three $\ll$ signs in $(\mathrm{P})$ are replaced by $\leq$ then this modified property $\left(\mathrm{P}_{<}\right)$also implies the quasimonotonicity of $f$ in $x$, under the assumptions of Theorem 1. Note that the converse is valid if, in addition, $E$ is a normed space and $f$ satisfies a local Lipschitz condition with respect to $x$; cf. [9].

\section{Another Characterization of QUASIMONotonicity}

To verify quasimonotonicity, it suffices to show (2) only for a few $\varphi \in K^{*}$ if these $\varphi$, regarded as supporting functionals of $K$, provide sufficiently many supporting points.

Theorem 2. Let $E$ be a Hausdorff topological vector space preordered by a cone $K$ with Int $K \neq \emptyset$. Let $S \subset K^{*}$ be such that the set

$$
\{x \in K: \varphi(x)=0 \text { for some nontrivial } \varphi \in S\}
$$

is dense in the boundary of $K$. Suppose $G \subset E$ is open, and $f: G \rightarrow E$ is continuous. If the implication (2) holds for all $x, y \in G$ and all $\varphi \in S$, then $f$ is quasimonotone increasing.

This result was motivated by Walter [11], [12, Theorem 12 XII] where the ordered Banach space $E=\ell_{\infty}(A)$ is underlying; cf. Remark 2 and [8, Beispiel 3].

Proof. By Theorem 1, it suffices to verify (P) rewritten for autonomous $f$. Suppose $v, w:\left[t_{0}, t_{1}\right] \rightarrow G\left(t_{0}<t_{1}\right)$ are differentiable functions which satisfy $v\left(t_{0}\right) \ll w\left(t_{0}\right)$ and

$$
v^{\prime}(t)-f(v(t)) \ll w^{\prime}(t)-f(w(t)) \quad\left(t_{0} \leq t \leq t_{1}\right) .
$$

Put

$$
u(t)=w(t)-v(t) \quad\left(t_{0} \leq t \leq t_{1}\right)
$$


To prove $u(t) \in \operatorname{Int} K$ for $t_{0} \leq t \leq t_{1}$, assume the contrary. Since $u\left(t_{0}\right) \in \operatorname{Int} K$, there exists $t_{2} \in\left(t_{0}, t_{1}\right]$ such that

$$
u(t) \in \operatorname{Int} K \quad\left(t_{0} \leq t<t_{2}\right)
$$

and such that $u\left(t_{2}\right)$ lies on the boundary of $K$.

Choosing some $t_{3} \in\left[t_{0}, t_{2}\right)$ sufficiently close to $t_{2}$, we may write

$$
f\left(w\left(t_{2}\right)\right)-f\left(v\left(t_{2}\right)\right) \ll \frac{u\left(t_{2}\right)-u\left(t_{3}\right)}{t_{2}-t_{3}},
$$

by (6). Since $G$ is open and $f$ is continuous, there is a neighborhood $\Delta$ of $0_{E}$ such that $w\left(t_{2}\right)+d \in G$ and

$$
f\left(w\left(t_{2}\right)+d\right)-f\left(v\left(t_{2}\right)\right) \leq \frac{u\left(t_{2}\right)+d-u\left(t_{3}\right)}{t_{2}-t_{3}}
$$

for all $d \in \Delta$. We now choose $d \in \Delta$ such that $u\left(t_{2}\right)+d$ is a member of the set (5). Consequently, $u\left(t_{2}\right)+d \in K$, and $\varphi\left(u\left(t_{2}\right)+d\right)=0$ for some nontrivial $\varphi \in S$. Substituting $u\left(t_{2}\right)+d=w\left(t_{2}\right)+d-v\left(t_{2}\right)$, we conclude that

$$
\varphi\left(f\left(v\left(t_{2}\right)\right)\right) \leq \varphi\left(f\left(w\left(t_{2}\right)\right)+d\right),
$$

by the restricted hypothesis (2). Thus the value of $\varphi$ at the left side of (8) is nonnegative, hence $0 \leq \varphi\left(u\left(t_{2}\right)+d\right)-\varphi\left(u\left(t_{3}\right)\right)$, and therefore $\varphi\left(u\left(t_{3}\right)\right) \leq 0$. But this contradicts $(7)$, and $(\mathrm{P})$ is verified.

Remark 2. Suppose $E$ is the real Banach space $\ell_{\infty}$ ordered by its natural strict cone

$$
K=\left\{x \in l_{\infty}: x_{n} \geq 0 \text { for all } n \in \mathbb{N}\right\},
$$

where $x=\left(x_{n}\right)_{n=1}^{\infty}$. Then Int $K \neq \emptyset$, and the set $S=\left\{\delta_{n}: n \in \mathbb{N}\right\}$ of all coordinate functionals $\delta_{n}: E \rightarrow \mathbb{R}, x \mapsto x_{n}$, satisfies the hypothesis of Theorem 2 .

We mention some other ordered Banach spaces consisting of functions $A \rightarrow \mathbb{R}$, such that their natural cone has interior points and such that the corresponding set of all evaluation functionals $x \mapsto x(\alpha)(\alpha \in A)$ may be taken in Theorem 2, similarly: $\ell_{\infty}(A)$ ( $A$ any set), $C^{k}(A)$ or $B V(A)(A \subset \mathbb{R}$ any compact interval having interior points, $k \in \mathbb{N}_{0}$ ), or $B C(A)$ ( $A$ any topological space).

Remark 3. The following example shows that we cannot omit the continuity condition on $f$ in Theorem 2. Put $E=\ell_{\infty}$ and $K, S$ as in Remark 2. Define $f: E \rightarrow E$ by $f(x)=\left(f_{n}(x)\right)_{n=1}^{\infty}$ where

$$
f_{n}(x)=1 \text { if } x_{n}=0 \text { and } x \in K, \quad f_{n}(x)=-2 \text { otherwise }
$$

$(n \in \mathbb{N}, x \in E)$. Then (2) holds for all $x, y \in E$ and all $\varphi \in S$. On the other hand, the functions $v, w:[0,2] \rightarrow E$,

$$
v(t)=0_{E}, \quad w(t)=(1+1 / n-t)_{n=1}^{\infty} \quad(0 \leq t \leq 2),
$$

satisfy $v(0) \ll w(0)$ and $v^{\prime}(t) \ll f(v(t))$ as well as $w^{\prime}(t) \gg f(w(t))$ for $0 \leq t \leq 2$. But even $v(t) \leq w(t)$ is false if $1<t \leq 2$. Thus $f$ cannot be quasimonotone increasing. This can also be verified by taking $x=0_{E}, y=(1 / n)_{n=1}^{\infty}$ and $\varphi$ as Banach limit in (2). 


\section{REFERENCES}

[1] E. Kamke, Zur Theorie der Systeme gewöhnlicher Differentialgleichungen II, Acta Math. 58 (1932), 57-85.

[2] R. Lemmert, Existenzsätze für gewöhnliche Differentialgleichungen in geordneten Banachräumen, Funkcial. Ekvac. 32 (1989), 243-249. MR 90i:34096

[3] M. Müller, Über das Fundamentaltheorem in der Theorie der gewöhnlichen Differentialgleichungen, Math. Z. 26 (1927), 619-645.

[4] S. Schmidt, Fixed points for discontinuous quasimonotone maps in sequence spaces, Proc. Amer. Math. Soc. 115 (1992), 361-363. MR 93c:47072

[5] A. Simon and P. Volkmann, Remark on quasimonotonicity, World Sci. Ser. Appl. Anal. 3 (1994), 543-548. MR 95h:34024

[6] A. Simon and P. Volkmann, Parabolic inequalities in ordered topological vector spaces, Nonlinear Analysis 25 (1995), 1051-1054. MR 96i:35142

[7] R. Uhl, An extension of Max Müller's theorem to differential equations in ordered Banach spaces, Funkcial. Ekvac. 39 (1996), 203-216. CMP 97:04

[8] P. Volkmann, Gewöhnliche Differentialungleichungen mit quasimonoton wachsenden Funktionen in topologischen Vektorräumen, Math. Z. 127 (1972), 157-164. MR 46:7661

[9] P. Volkmann, Über die Invarianz konvexer Mengen und Differentialungleichungen in einem normierten Raume, Math. Ann. 203 (1973), 201-210. MR 48:667

[10] P. Volkmann, Cinq cours sur les équations différentielles dans les espaces de Banach, A. Granas and M. Frigon (eds.), Topological methods in differential equations and inclusions, Kluwer, Dordrecht, 1995, 501-520. MR 96k:34138

[11] W. Walter, Gewöhnliche Differential-Ungleichungen im Banachraum, Arch. Math. 20 (1969), 36-47. MR 39:5908

[12] W. Walter, Differential and integral inequalities, Springer, Berlin, 1970. German edition 1964. MR 42:6391

Mathematisches Institut II, Universität Karlsruhe, D-76128 Karlsruhe, Germany

E-mail address: roland.uhl@math.uni-karlsruhe.de 